\title{
Evaluierung von Stillproblemen bei einem Zungenbändchen
}

Sara-Maria Simon

\author{
Misserfolg beim Stillen stellt ein häufiges Problem auf Wochenstationen dar. Die \\ Rolle eines Zungenbändchens bzw. einer Ankyloglossie als Stillhindernis wird in \\ der wissenschaftlichen Literatur und im klinischen Alltag kontrovers diskutiert. \\ Eine richtige Diagnose und zeitnahe Therapie können Müttern und Neugebore- \\ nen größere Stillprobleme ersparen. Daher sollte dieses relevante und behandel- \\ bare Stillhindernis in den Geburtskliniken und pädiatrischen Vorsorgeunter- \\ suchungen berücksichtigt werden.
}

\section{Grundlagen zur Ankyloglossie und zum Stillen \\ Definition Ankyloglossie}

Ein Zungenbändchen (Frenulum linguae) spannt sich als dünnes, teilweise auch fibröses Band zwischen Mundboden und Zungengrund ( $\triangleright$ Abb.1). Bei einer Ankyloglossie handelt es sich um eine angeborene Entwicklungsstörung der Zunge. Dabei wird die Zunge durch ein zu kurzes oder zu straffes Zungenbändchen am Mundboden fixiert. Dieses restriktive Zungenbändchen setzt häufig zu weit vorne an der Zungenspitze und an der Zahnleiste an [1].

Des Weiteren gibt es auch dorsale Zungenbändchen, die bei der Inspektion wenig auffällig erscheinen. Bei der Palpation können sich diese jedoch als verdicktes oder submuköses Frenulum darstellen, das die Beweglichkeit an der Basis der Zunge behindert. Die posteriore Form ist dadurch schwieriger zu diagnostizieren $[1,2]$.

Nach der rein morphologischen Bewertung eines restriktiven Zungenbändchens sollten auch funktionelle Kriterien berücksichtigt werden, die ein erfahrener Untersucher anhand der eingeschränkten Mobilität der Zunge erkennt [3].
Merke

Bei der Inspektion des Mundes sieht man immer einen Zungenbändchenrest, im medizinischen Alltagsgebrauch ist mit dem Begriff „Zungenbändchen“ jedoch häufig bereits das „restriktive Zungenbändchen" gemeint.

\section{PRAXIS}

Woran erkenne ich ein auffälliges Zungen-

bändchen?

- leichte Kerbe an der Zungenspitze oder herzförmige Zungenspitze

- kranialer Ansatz des Frenulums an oder nahe der Zungenspitze

- kaudaler Ansatz des Frenulums an oder auf der unteren Zahnleiste

\section{Embryologie}

Die Zungenentwicklung findet zwischen der 4. und der 8. Schwangerschaftswoche statt. Initial ist die Zunge fest mit dem Mundboden verwachsen. Während der Ablösung des vorderen Teils der Zunge vom Mundboden bleibt das Zungenbändchen als einziges Überbleibsel der ursprünglichen Anheftung in der Mittellinie an der Unterseite der Zunge bestehen. Störungen in dieser Phase der Apoptose verursachen ein restriktives Zungenbändchen oder eine Ankyloglossie [4]. 


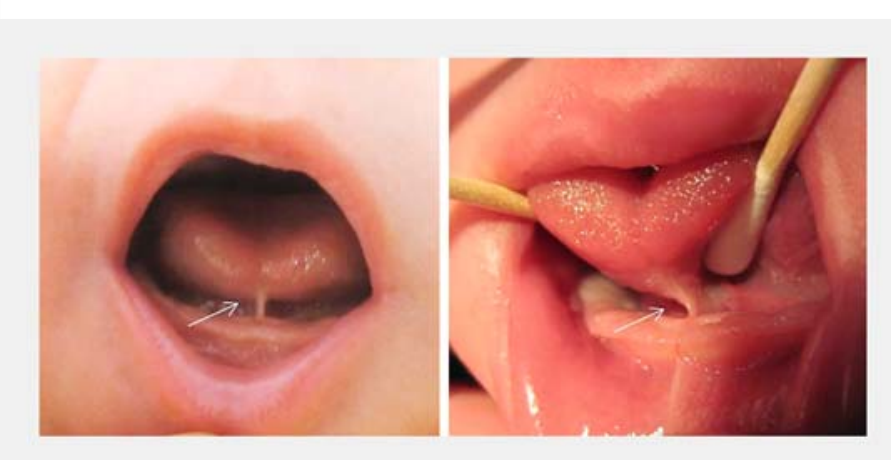

- Abb. 1 Erscheinungsbild einer Ankyloglossie beim Säugling.

\section{FALLBEISPIEL}

\section{Fall 1}

Ein weibliches Neugeborenes $(40+2$ T SSW) wird spontan entbunden und direkt in den ersten Lebensstunden angelegt. Die Mutter, eine 24-jährige Erstgravida, berichtet am 2. Lebenstag von Stillproblemen. Das Kind nehme die Brustwarze nicht gut und rutsche beim Trinken immer wieder ab. Der Mutter sei außerdem ein lautes Schnalzen beim Saugen aufgefallen. In der Stillberatung fällt auf, dass das Kind die Zunge nicht über die Unterlippe hinausstrecken kann und die peristaltische Welle beim Saugen hinter der Zungenspitze abflacht. Die Verdachtsdiagnose lautet Ankyloglossie.

Das Neugeborene wird daher zur Mitbeurteilung in die MundKiefer-Gesichtschirurgie überwiesen. Dort wird das Frenulum problemlos durchtrennt. Direkt im Anschluss wird das Neugeborene erneut angelegt. Das Stillen funktioniert nun deutlich besser. Die Mutter berichtet, dass die Brust besser stimuliert werde und ist erleichtert, den kurzen und einfachen Eingriff durchführen lassen zu haben.

\section{Risikofaktoren des Stillens}

Da das Stillen zahlreiche gesundheitliche Vorteile für Mutter und Säugling bietet, empfiehlt die Weltgesundheitsorganisation (WHO) allen Müttern das ausschließliche Stillen ihrer Kinder in den ersten 6 Lebensmonaten [5]. Nach einer initialen Stillquote von 85-87\% werden die Empfehlungen der WHO aktuell jedoch nur in $11-40 \%[6,7]$ umgesetzt. Dem frühzeitigen Abrechen des Stillens gehen meistens Stillschwierigkeiten voraus.

Eine unzureichende Milchaufnahme ist der häufigste Grund für ein frühzeitiges Beenden des Stillens. Sehr selten ist eine unzureichende mütterliche Milchproduktion die tatsächliche Ursache dafür, z.B. aufgrund von mütterlichen Erkrankungen, endokrinen Störungen oder laktationshemmenden Medikamenten [8,9]. Viel häufiger entsteht der mangelhafte Milchtransfer jedoch durch ein gestörtes Saugverhalten des Säuglings, z. B. bei einem schlechten Andocken oder Schläfrigkeit des Säuglings in den ersten Tagen. Der unzurei- chende Milchtransfer hat einen Circulus vitiosus zur Folge, wobei die fehlende Energiezufuhr zu einem reduzierten Trinkverhalten und letztlich auch zu einem reduzierten Milchtransfer führt [10].

Schmerzen an Brust und Brustwarzen sind der zweithäufigste Grund für ein vorzeitiges Abstillen. Ein unzureichendes Andocken des Säuglings zu Beginn des Stillens verursacht vielfach wunde Brustwarzen mit Einblutungen, Rhagaden oder Blasen, was oft durch ungünstige Stillpositionen, ungenügende Mundöffnung des Kindes oder das Hinauszögern von Stillmahlzeiten begünstigt wird. Orale Fehlbildungen, wie z. B. Spaltbildungen (Lippe und/oder Kiefer und/oder Gaumen) oder eine Ankyloglossie bei den Säuglingen sind eine weitere Ursache für wunde Brustwarzen $[11,12]$.

\section{Merke \\ Häufig entstehen Stillprobleme und Schmerzen an Brust und Brustwarzen durch ein ungenügendes Saugverhalten des Säuglings.}

Weitere relevante prognostische Faktoren des Stillerfolgs sind eine fehlende Stillerfahrung, Untergewicht oder Frühgeburtlichkeit des Säuglings sowie ein Kaiserschnitt statt einer Spontangeburt, wie u. a. Ergebnisse einer multivariaten Analyse des Freiburger Geburtenkollektivs von 2014-2015 zeigten [13]. Ein erfolgreiches Stillen kann durch weitere Variablen wie z. B. eine lange Geburtsdauer >14 Stunden, frühes Zufüttern, Verwendung von Flaschenkost und Schnullern, flache oder eingezogene Brustwarzenformen, mütterliches Übergewicht $\left(B M I>27 \mathrm{~kg} / \mathrm{m}^{2}\right)$ oder Zweifel an der Milchmenge negativ beeinflusst werden $[10,14]$.

\section{Saugmechanismus}

\section{Merke}

Das erfolgreiche Stillen basiert auf einem komplexen Zusammenspiel von Mutter und Kind, sowie der korrekten kindlichen Koordination von Saugen, Schlucken und Atmen.

Eine erstgebärende Mutter muss die korrekte Anlegetechnik beim Stillen zunächst lernen. Hingegen wird dem Neugeborenen die instinktive Nahrungsaufnahme durch frühkindliche Reflexe erleichtert. Durch eine Stimulation der Mundregion wird der „Suchreflex“ ausgelöst. Daraufhin dreht der Säugling den Kopf in Richtung der Berührung und öffnet gleichzeitig den Mund, um an die Brust anzudocken. Durch die Berührung des Gaumens wird der „Saugreflex“ initiiert. Er ermöglicht zusammen mit dem "Schluckreflex“ die erfolgreiche Nahrungsaufnahme an der Brust [15]. 
Ein nutritives Saugen besteht aus einem rhythmischen Wechsel zwischen Saugen und Auspressen der Brustwarze. Dabei wird die Brustwarze vollständig mitsamt Warzenvorhof in die Mundhöhle aufgenommen. Der Zunge kommt dabei eine besondere Bedeutung während des Saugmechanismus zu. Sie muss während des Saugens folgende Bewegungen ausführen:

- eine Vorwärtsbewegung,

- die Ausbreitung in seitlicher Richtung,

- das Verdünnen der Zungenspitze vor der Bildung des Saugschlusses um die Brustwarze,

- das Anheben der Zungenspitze,

- eine Wellenbewegung in Richtung Rachen.

Gleichzeitig werden der Unterkiefer angehoben und die Milchgänge komprimiert. Dabei findet eine „Melkdruckbewegung “ statt, d. h. die Brustwarze wird gegen den harten Gaumen gepresst und dadurch Milch ausgepresst, die sich im Mund-Rachen-Raum sammelt. Zur Auslösung des Schluckreflexes wird der hintere Teil der Zunge gegen den weichen Gaumen gedrückt, wodurch sich die Gaumensegel heben und die NasenRachen-Höhle abdichten. Zuletzt sinkt der hintere Teil der Zunge ab, wodurch ein Vakuum erzeugt wird. Dieses bewirkt zum einen, dass sich die Brustwarze erneut mit Milch füllt, außerdem wird sie dadurch weiter in die Mundhöhle gezogen [15].

\section{Merke}

Eine eingeschränkte Zungenbeweglichkeit, z. B. durch eine Ankyloglossie, kann zu einem gestörten Saugmechanismus und zu Stillproblemen führen.

\section{Ankyloglossie als Stillrisiko}

Ein straffes, kurzes Zungenbändchen wird mit folgenden funktionellen Stillproblemen assoziiert [16]:

- mütterliche Brustwarzendefekte,

- Brustschmerzen,

- geringer Milchfluss,

- Gedeihstörungen,

- Verweigerung der mütterlichen Brust.

\section{Merke}

Als wesentlicher Mechanismus für Stillprobleme bei Ankyloglossie gilt ein unzureichendes Andocken an der Brust $[17,18]$.

Neugeborene mit einer Ankyloglossie und eingeschränkter Zungenmobilität sind nicht in der Lage, einen ausreichenden Saugschluss zu bilden. Um beim Stillen die Brust im Mund zu halten, wird somit der eigene Kiefer und das Wangenfettpolster genutzt [16]. Dies kann zu starken Schmerzen und Verletzungen der Brustwarzen führen. Die eingeschränkte Vorwärtsbewegung der Zunge beeinträchtigt den Start der peristaltischen Welle. Um ein Vakuum herzustellen, wird häufig die Unterlippe eingezogen. Schnalzgeräusche und ein häufiges Loslassen deuten auf ein unvollständiges Vakuum hin.

Da Brustwarze und Brustwarzenhof nicht vollständig eingesaugt werden können, wird die Melkdruckbewegung auf die Milchgänge behindert [19]. Daraus folgen wiederum ein mangelhafter Milchtransfer und ggf. ein daraus resultierender Gewichtsverlust des Säuglings [17]. Außerdem kann ein Milchstau entstehen, wenn die Brust nicht vollständig entleert oder nicht optimal stimuliert wird, sodass keine ausreichende Milchmenge produziert werden kann. Folge davon können ausgedehnte Stillzeiten sowie eine häufige Umstellung auf Flaschenernährung sein [19].

\section{Merke \\ Hinweise auf einen gestörten Saugmechanismus fin- den sich bei einer Ankyloglossie durch ein starkes Zusammenpressen des Kiefers, Einziehen der Unter- lippe, Schnalzgeräusche beim Saugen oder ein häufi- ges Loslassen der Brust. \\ Epidemiologie von Ankyloglossie und Stillproblemen}

In der wissenschaftlichen Literatur variiert die Inzidenz von Ankyloglossie zwischen 1 und 15\% [16]. Die breite Streuung kann durch das Fehlen diagnostischer Standards sowie einer einheitlichen Definition der Ankyloglossie erklärt werden. Männliche Neugeborene sind häufiger betroffen, wie das Geschlechterverhältnis von $1,5: 1$ bis $2,6: 1$ in entsprechenden Studien zeigt [17, $18]$.

Auch die Inzidenz von Stillproblemen bei Säuglingen mit Ankyloglossie schwankt zwischen 25 und $80 \%$ [18, 20]. Im Freiburger Geburtenkollektiv traten zwischen 2014 und 2015 bei etwas mehr als der Hälfte aller Neugeborenen mit restriktivem Zungenbändchen (55\%) Stillprobleme auf, signifikant häufiger als bei Neugeborenen ohne restriktives Zungenbändchen, deren Mütter nur in $42 \%$ Stillprobleme angaben [13].

\footnotetext{
Merke

Insbesondere schwere Stillprobleme sind bei Ankyloglossie mit $29 \%$ signifikant häufiger als bei einem „normalen“ Zungenbändchen (14\%) [13].
} 


\section{Diagnostik}

\section{Klassifikation der Ankyloglossie: das „Hazel- baker Assessment Tool for Lingual Frenulum Function“ (ATLFF) als Screeningtool}

Die objektive Bewertung des Zungenbändchens kann mithilfe des „Hazelbaker Assessment Tool for Lingual Frenulum Function“ (ATLFF) durchgeführt werden. Das Tool wurde von Alison Hazelbaker 1993 entwickelt und bewertet neben Kriterien zum Erscheinungsbild von Zunge und Zungenbändchen auch die Funktion der Zungenbeweglichkeit. Jede Untereinheit des Scores wird mit 0 bis 2 Punkte bewertet, wobei maximal 10 Punkte für ein unauffälliges Erscheinungsbild sowie 14 Punkte für eine perfekte Funktion der Zungenbeweglichkeit vergeben werden können [21] (\Abb. 2).

Nachfolgend werden die Untereinheiten des ATLFFScores mit der zugehörigen Untersuchungstechnik erklärt.

\section{Erscheinungsbild}

Das Erscheinungsbild wird durch das Anheben der Zunge mit einer Hohlsonde untersucht ( $\downarrow$ Abb.3). Dabei untersucht man die Form der Zungenspitze, die Länge und Elastizität des Zungenbändchens bei angehobener Zunge. Außerdem wird der Ansatz des Zungenbändchens an der unteren Zahnleiste und der Zunge bewertet.

\section{Funktion}

- Lateralisation (Seitbewegung der Zunge): Der „transversale Zungenreflex“ wird ausgelöst, indem der Finger des Untersuchers entlang der Zahnleiste bis an die lateralen Zungenränder streicht. Die Zungenspitze folgt dadurch dem Finger nach rechts und links. Auf diese Weise kann die Seitbewegung der Zunge beurteilt werden.

- Anheben der Zunge: Dies kann zum einen beim schreienden Neugeborenen oder durch ein vorsichtiges Hochdrücken der Zunge mit dem Finger bei geöffnetem Mund beobachtet werden.

- Extension (Herausstrecken der Zunge): Streicht man die Unterlippe vertikal in Richtung Kinn aus, wird ein „Extensionsreflex" ausgelöst. So kann das Herausstrecken der Zunge erfasst werden.

- Ausbreitung der Zunge: Dieser Parameter beschreibt, wie sich die Zunge zu Beginn des Saugschlusses gleichmäßig verdünnt und nach vorne ausbreitet.

- Saugschluss und Peristaltik: Durch die Berührung der Lippen in einer Auf- und Abwärtsbewegung wird der „Suchreflex“ ausgelöst. Das Neugeborene öffnet daraufhin den Mund, eine Berührung des Gaumens mit dem kleinen Finger löst den „Saugreflex“ aus.
Dadurch werden gleichzeitig Saugschluss und Peristaltik beurteilt.

- Beim Item Saugschluss wird beschrieben, wie eng sich die Zunge um den Finger legt.

- Für die Peristaltik wird die Wellenbewegung der Zunge, die von der Zungenspitze ausgeht, bewertet.

- Zurückschnalzen der Zunge: Ein unfreiwilliges Zurückziehen der Zunge während des Saugens erzeugt schnalzende Geräusche. Diese werden während des Saugens und durch zusätzliches Befragen der Mutter beurteilt.

\section{Merke}

Fast alle Parameter des ATLFF-Funktion-Scores sind auch Teil des korrekten Saugmechanismus eines Säuglings.

\section{Therapie}

Der natürliche Verlauf eines unbehandelten Zungenbändchens ist umstritten. Manche Autoren vermuten, dass dieses durch eine zunehmende Dehnung und Beanspruchung wachsen könne [22]. Andere, wie A. Hazelbaker, nehmen an, dass sich das Zungenbändchen zwar proportional zum Zungenwachstum verlängere, die Restriktion der Zunge jedoch immer persistiere [21].

\section{FALLBEISPIEL}

Fall 2

Ein männliches Neugeborenes (38+3 T SSW) kommt per primärer Sectio auf die Welt. Am 4. Lebenstag berichtet die 32-jährige Zweitgravida von Stillproblemen. Sie habe starke Schmerzen und bereits blutige Brustwarzen. Obwohl sie ihr erstes Kind erfolgreich gestillt habe, überlege sie, abzustillen, da sie die Schmerzen kaum aushalte. In der Untersuchung der Mundhöhle im Rahmen der U2 fällt auf, dass die Zungenspitze auffällig herzförmig ist und das Neugeborene beim Saugen vermehrt den Kiefer zusammenpresst. Die Verdachtsdiagnose lautet Ankyloglossie.

Das Neugeborene wird zur Mitbeurteilung in die Mund-Kiefer-Gesichtschirurgie überwiesen. Dort wird das Frenulum durchtrennt. In den Tagen danach zeigt sich eine Besserung der wunden Brustwarzen, und die Mutter berichtet über deutlich geringere Schmerzen beim Stillen. 


\begin{tabular}{|c|c|c|}
\hline $\begin{array}{l}\text { Screeningbogen } \\
\text { zur Funktion des Zungenbandes } \\
\text { (ATLFF-Assessment Tool for Lingual Frenulum Function) } \\
\text { (C) Alison K. Hazelbaker } 2017\end{array}$ & $\begin{array}{l}\text { Name der Mutter: } \\
\text { Name des Babys: } \\
\text { Alter des Babys: } \\
\text { Datum der Untersuchung: }\end{array}$ & 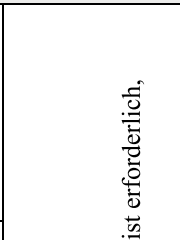 \\
\hline \multicolumn{2}{|l|}{ KRITERIEN ZUR FUNKTION } & \\
\hline $\begin{array}{l}\text { Lateralisation } \\
2 \text { vollständig } \\
1 \text { Zungenkörper, aber nicht die Zungenspitze } \\
0 \text { keine } \\
\text { Anheben der Zunge } \\
2 \text { Zungenspitze bis zur halben Höhe des Mundes } \\
1 \text { nur die Ränder bis zur halben Höhe des Mundes } \\
0 \text { Zungenspitze bleibt bei der Oberkante der unteren } \\
\text { Zahnleiste ODER Zungenspitze geht nur beim Schließen } \\
\text { der Kiefer nach oben UND/ODER Grübchen in der } \\
\text { Zungenmitte } \\
\text { Herausstrecken der Zunge } \\
2 \text { Zungenspitze über der Unterlippe } \\
1 \text { Zungenspitze nur über der unteren Zahnleiste } \\
0 \text { keiner dieser ODER Buckelbildung der vorderen Zunge } \\
\text { oder der Zungenmitte UND/ODER Grübchen } \\
\text { Die Zunge breitet sich nach vorne aus und wird dünn } \\
2 \text { vollständig } \\
1 \text { mäßig ODER teilweise } \\
0 \text { kaum ODER gar nicht }\end{array}$ & $\begin{array}{l}\text { Saugschluss } \\
2 \text { starker Saugschluss, Zunge umschließt mit ganzem } \\
\text { Zungenrand } \\
1 \text { Zunge hält nur mit den seitlichen Zungenrändern ODER } \\
\text { mäßiger Saugschluss } \\
0 \text { schwacher ODER kein Saugschluss } \\
\text { Peristaltik } \\
2 \text { vollständig von vorne nach hinten, beginnend an der } \\
\text { Zungenspitze } \\
1 \text { schwache Peristaltik ODER Peristaltik beginnt hinter der } \\
\text { Zungenspitze } \\
0 \text { keine ODER Peristaltik in umgekehrter Richtung } \\
\text { Zurückschnalzen der Zunge } \\
2 \text { nie } \\
1 \text { ab und zu } \\
0 \text { häufig ODER bei jeder Saugbewegung }\end{array}$ & 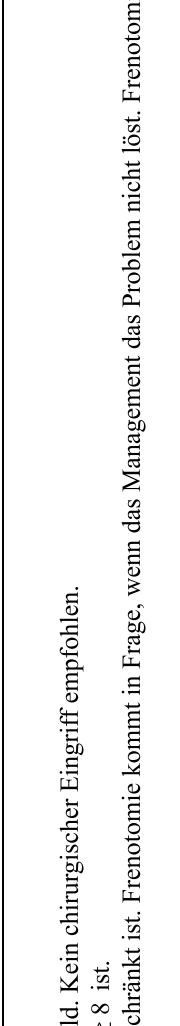 \\
\hline \multicolumn{2}{|l|}{ KRITERIEN ZUM ERSCHEINUNGSBILD } & \\
\hline $\begin{array}{l}\text { Erscheinungsbild der Zunge, wenn angehoben } \\
2 \text { rund ODER eckig } \\
1 \text { leichte Kerbe an der Zungenspitze zu sehen } \\
0 \text { herzförmige Zungenspitze } \\
\text { Länge des Zungenbandes bei angehobener Zunge } \\
2 \text { länger als } 1 \mathrm{~cm} \text { ODER Zungenband fehlt } \\
11 \mathrm{~cm} \text { lang } \\
0 \text { kürzer als } 1 \mathrm{~cm} \\
\text { Ansatz des Zungenbandes an der unteren Zahnleiste } \\
2 \text { am Mundboden ODER deutlich unter der Oberkante der } \\
\text { Zahnleiste (deutlich unter dem Alveolarkamm) } \\
1 \text { direkt unter der Oberkante der Zahnleiste } \\
0 \text { an der Oberkante der Zahnleiste }\end{array}$ & $\begin{array}{l}\text { Elastizität des Zungenbandes } \\
2 \text { sehr elastisch (ausgezeichnet) } \\
1 \text { mäßig elastisch } \\
0 \text { wenig ODER überhaupt nicht elastisch } \\
\text { Ansatz des Zungenbandes an der Zunge beansprucht } \\
2 \text { weniger als } 50 \% \text { der Mittellinie der Zungenunterseite } \\
150-75 \% \text { der Mittellinie der Zungenunterseite } \\
075-100 \% \text { der Mittellinie der Zungenunterseite }\end{array}$ & 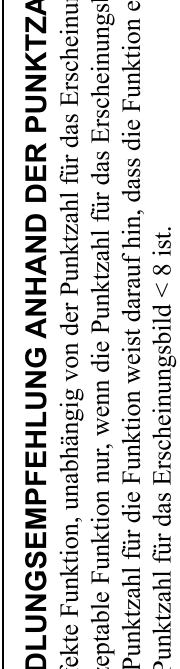 \\
\hline \multicolumn{2}{|l|}{ PUNKTZAHL } & \\
\hline Punktzahl Funktion: __ Punktzahl Erscheinung & Kombinierte Punktzahl: ___ & 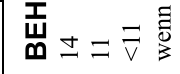 \\
\hline
\end{tabular}

Übersetzung: Márta Guóth-Gumberger, IBCLC; Rückübersetzung: Elizabeth Hormann, IBCLC; genehmigt: Dr. Alison Hazelbaker, 2015

- Abb.2 Screeningbogen zur Funktion des Zungenbändchens. (ATLFF - Assessment Tool for Lingual Frenulum Function) $\odot$ Alison K. Hazelbaker 2014. Übersetzung: Márta Guóth-Gumberger, IBCLC; Rückübersetzung: Elizabeth Hormann, IBCLC; genehmigt: Dr. Alison Hazelbaker, 2015 [rerif] 


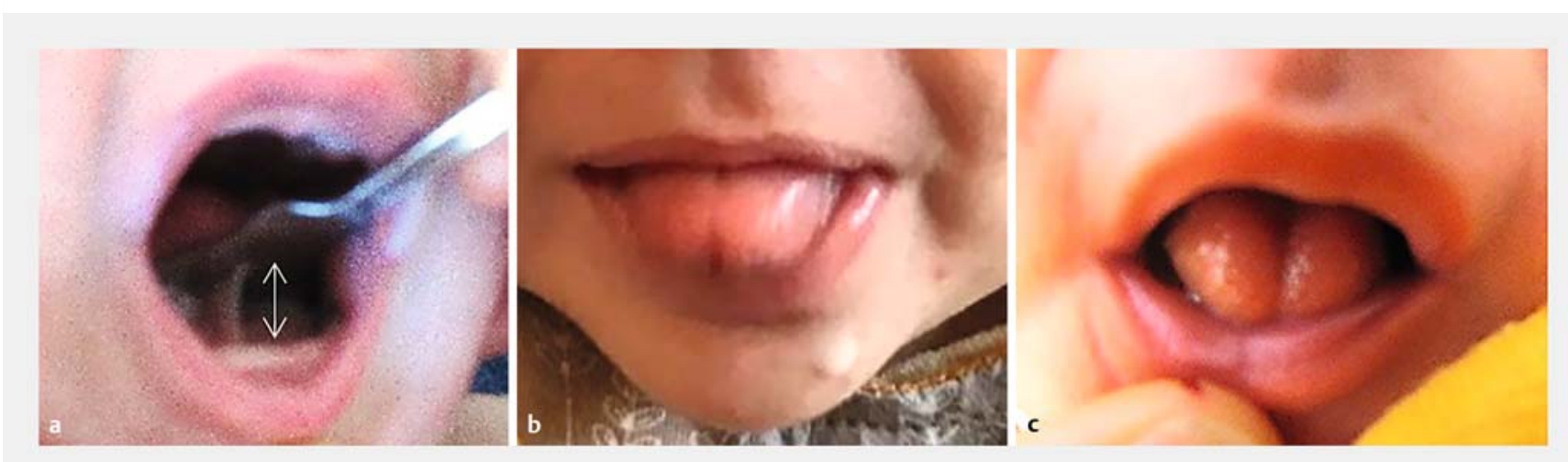

- Abb. 3 Kriterien des ATLFF-Scores („Hazelbaker Assessment Tool for Lingual Frenulum Function“) zum Erscheinungsbild der Zunge. a Untersuchung des Zungenbändchens mit einer Hohlsonde, Darstellung der Länge des Zungenbändchens. b Form der Zungenspitze: Zungenspitze mit Kerbe. c Herzförmige Zungenspitze.

\section{Operative Verfahren}

Stillprobleme im Säuglingsalter, später auch Artikulationsprobleme sowie mechanische oder soziale Probleme können Indikationen für eine operative Therapie sein [22].

Merke

Eine operative Therapie der Ankyloglossie soll die Zungenbeweglichkeit verbessern.

Am häufigsten wird bei Säuglingen eine Frenulotomie oder Frenotomie, d.h. die Durchtrennung des dünnen, membranösen Zungenbändchens angewandt (s. - Abb. 4a sowie Fallbeispiel 1 u. 2). In der Regel wird diese Methode ohne Lokalanästhesie durchgeführt. Dabei wird das Zungenbändchen mithilfe einer sterilen Schere durchtrennt, sie kann jedoch auch durch eine Laserablation durchgeführt werden $[17,22,23]$.

Eine weitere operative Methode ist die Frenuloplastie (s. Abb.4b). Diese umfasst die vertikale oder horizontale Durchtrennung des Zungenbändchens mit anschließender plastischer Rekonstruktion der betroffenen Strukturen. Diese Methode wird unter Allgemeinanästhesie und vorrangig bei älteren Kindern oder komplexen Fehlbildungen angewandt [24].
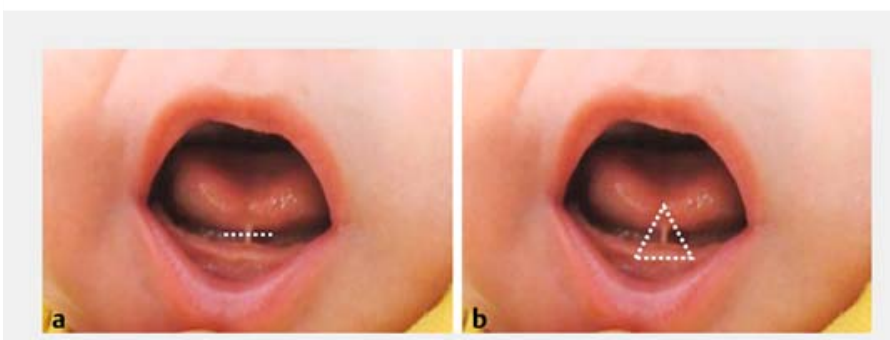

Abb.4 Operative Techniken zur Durchtrennung des Zungenbändchens. a Frenulotomie. b Frenuloplastie.
Komplikationen, wie minimale und kurze Blutungen, treten laut Studienergebnissen selten bei den Eingriffen auf [25]. Auch Schmerzen, Begleitverletzungen oder Rezidive mit der Notwendigkeit für Reoperationen sind sehr selten $[12,20,26,27]$.

Merke

Trotz des schnellen, einfach erscheinenden Eingriffs und der geringen Komplikationsrate sollte eine Frenulotomie von qualifizierten Ärzten, z. B. MundKiefer-GesichtschirurgInnen oder HNO-ÄrztInnen, durchgeführt werden.

\section{Studienlage}

Die Effizienz sowie die Indikationsstellung einer Operation bei einer Ankyloglossie sind wissenschaftlich weiterhin umstritten. Der Großteil der Untersuchungen, darunter auch randomisiert-kontrollierte Studien, die mögliche Outcomes bezüglich einer Verbesserung der Stilldauer, -qualität oder Zungenmobilität erforschten, wurden mit einem „ungenügenden Evidenzgrad“ bewertet. Mit einem „niedrigen Evidenzgrad“ wurden lediglich der positive Effekt einer Operation auf Brustschmerzen und Stillerfolg ausgezeichnet [1]. Unklar bleiben langfristige Folgen und der Einfluss auf objektive Outcomes, z. B. auf Stillskalen [28]. Somit besteht für den positiven Effekt einer Frenulotomie aufgrund bedeutsamer Limitation der Studien bisher maximal ein „niedriger Evidenzgrad“. Aus diesem Grund werden weitere größere Studien sowie klare Diagnostikkriterien gefordert, um eine eindeutige Empfehlung auszusprechen $[1,24,29]$.

Unserer Erfahrung nach profitieren diejenigen Neugeborenen mit restriktivem Zungenbändchen mit einem niedrigen ATLFF-Funktions-Score sowie signifikanten Stillproblemen von einer frühzeitigen Frenulotomie. Diese Neugeborenen hatten im Follow-up nach ca. 14 
Tagen signifikant weniger Stillprobleme als diejenigen, bei denen keine Frenulotomie durchgeführt wurde. Diejenigen Neugeborenen mit Ankyloglossie ohne jegliche Stillprobleme benötigen jedoch keine Intervention [13].

\section{Merke}

Eine Frenulotomie erfordert eine gezielte Indikationsstellung.

\section{Evaluierung von Stillproblemen bei Ankyloglossie}

- Wie kann man im klinischen Alltag durch ein frühzeitiges Screening relevanter Risikofaktoren den Stillerfolg verbessern?

- Woran erkennt man, welcher Säugling mit Ankyloglossie ein erhöhtes Risiko für Stillprobleme hat?

Stillprobleme treten bei etwas mehr als der Hälfte aller Neugeborenen mit Ankyloglossie auf. Da eine Ankyloglossie ein behandelbares Stillhindernis darstellt, sollte die Ankyloglossie unbedingt als solches berücksichtigt werden. Stillprobleme können dabei primär durch die Funktionseinschränkung der Zunge induziert sein, die Bedeutung der Zungenbeweglichkeit für einen erfolgreichen Saugmechanismus ist einleuchtend.

Gleichzeitig können Stillprobleme bei Säuglingen mit Ankyloglossie aber auch multifaktoriell bedingt sein. Die Berücksichtigung multipler Risikofaktoren bildet den Stillerfolg bei Ankyloglossie nach unseren Daten daher besser ab als die alleinige Bewertung der Ankyloglossie. Ein Screeningtool, welches neben dem Zungenbändchen-spezifischen ATLFF-Score auch weitere Stillhindernisse miteinschließt, wies nach unseren Daten eine höhere Sensitivität und Spezifität auf als die Bewertung des Zungenbändchens mit dem ATLFFScore allein ( $\mathbf{A b b} \mathbf{5} \mathbf{5}$ ).

- Abb. 6 zeigt auf der Grundlage dieser Daten ein mögliches Screeningtool. Dieses könnte es dem medizinischen Personal in Geburtskliniken und in der ambulanten postpartalen Betreuung erleichtern,

- Stillrisiken und Stillprobleme früher zu erkennen,

- durch gezielte Stillförderungsmaßnahmen das Stillverhalten zu verbessern und

- in indizierten Fällen eine frühzeitige Intervention einzuleiten.

In einem ersten Schritt sollten hierfür bereits im Rahmen der U1-Vorsorgeuntersuchung mögliche Risikofaktoren für Stillprobleme erfasst werden (s. > Tab. 1). Gleichzeitig sollte bei der Untersuchung der Mundhöhle ein erster Blick auf das Zungenbändchen geworfen werden. Trifft einer dieser Risikofaktoren zu oder ist

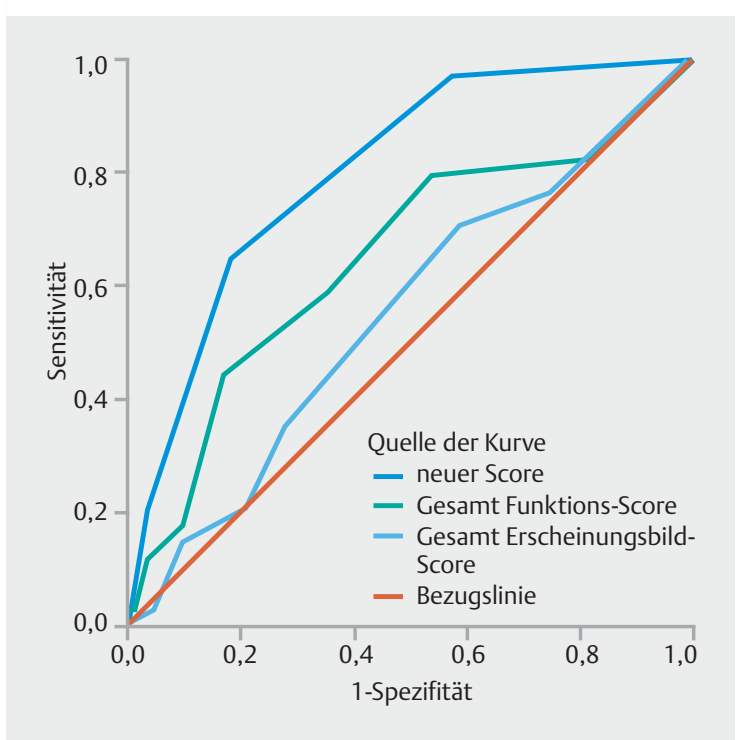

Abb.5 Vorhersage des Stillerfolgs bei Ankyloglossie mithilfe verschiedener Scores (ROC = Kurve zum Outcome: "schwere Stillprobleme“; AUC = Area under the Curve = neuer Score $0,8^{* * *}$; Gesamt Funktions-Score $0,6^{*}$, Gesamt Erscheinungsbild-Score $0,5^{*} ; n=116 ;{ }^{*}: p \leq 0,05,{ }^{* *}$ : $\left.p \leq 0,01,{ }^{* * *}: p \leq 0,001\right)$. Der Gesamt-Funktions-Score und der Gesamt-Erscheinungsbild-Score sind Teile des ATLFFScores ( $\triangleright$ Abb. 2). Der neue Score (s. a. $\triangleright$ Abb. 6) umfasst folgende Faktoren: fehlende Stillerfahrung, Untergewicht des Säuglings, Gesamt Funktions-Score < 11 Punkte im ATLFF (Assessment Tool for Lingual Frenulum Function), pathologische Peristaltik (Untereinheit des ATLFF-Funktions-Scores) (s. $>$ Tab. 1 und $\gg$ Tab. 2 ).

(Quelle: S.-M. Schlatter: Zungenbändchen und Stillerfolg - eine prospektive Studie. Dissertation, Medizinische Fakultät der Albert-Ludwigs-Universität Freiburg; 2018).

das Zungenbändchen morphologisch auffällig, sollte frühzeitig eine Stillberatung in die Wege geleitet werden.

\section{Merke}

Durch eine frühzeitige Stillberatung könnten Stillprobleme aufgrund von äußeren Faktoren, wie z. B. einer fehlenden Stillerfahrung, vorgebeugt bzw. abgemildert werden.

Spätestens bei der U2-Vorsorgeuntersuchung sollten dann gezielte Stillprobleme thematisiert werden. Dabei sollten mögliche Probleme beim Andocken, Schmerzen beim Stillen sowie wunde Brustwarzen erfragt werden. Im Rahmen der körperlichen Untersuchung sollte der ATLFF-Score zur Bewertung der Zungenbeweglichkeit und des Saugmechanismus des Neugeborenen angewendet werden ( $>$ Tab.2, $\triangleright$ Abb.6).

Eine Gesamtpunktzahl des ATLFF-Funktions-Scores $<11$ Punkten sowie eine pathologische Peristaltik der Zunge sprechen dabei für eine relevante Funktionsstörung $(\triangleright$ Tab.2). Diese Kohorte profitiert bei signifikanten Stillproblemen insbesondere trotz erfolgter Stillbera- 


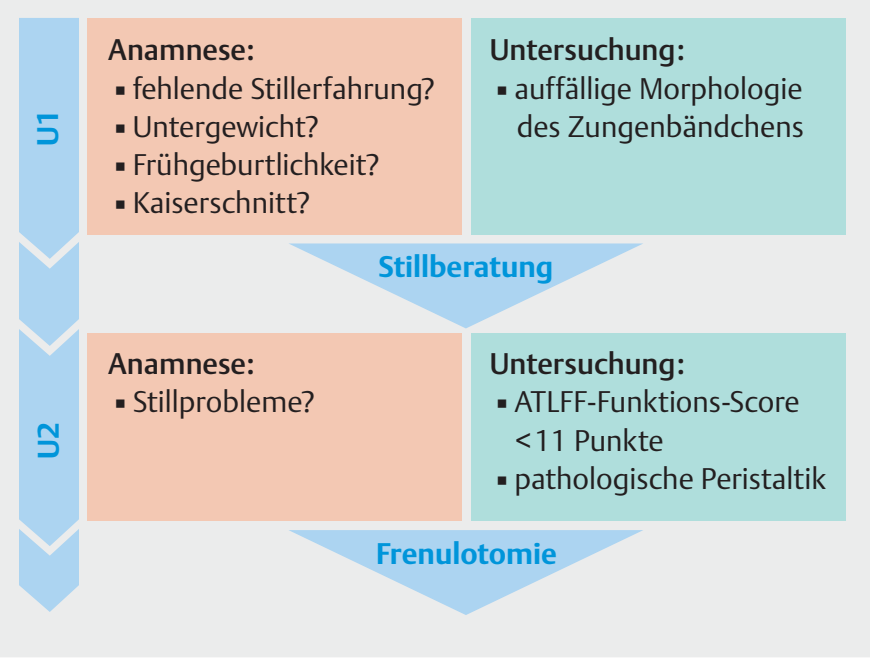

- Abb. 6 Screeningtool zur Erfassung von Stillrisiken und zur Vorbeugung von Stillproblemen (ATLFF = Assessment Tool for Lingual Frenulum Function).

- Tab. 1 Screening bei der U1: Mögliche Risikofaktoren.

\begin{tabular}{|l|l|}
\hline Variable & Ausprägung \\
\hline \multirow{2}{*}{ Stillerfahrung } & ja \\
\hline \multirow{2}{*}{ Untergewicht } & nein \\
\hline Frühgeburtlichkeit & Gewicht $>2500 \mathrm{~g}$ \\
\hline \multirow{2}{*}{ Ankyloglossie } & Gewicht $\leq 2500 \mathrm{~g}$ \\
\hline & $\geq 37+0$ T SSW \\
\hline Kaiserschnitt & $<37+0$ T SSW \\
\hline & nein \\
\hline & ja \\
\hline & nein \\
\hline & ja \\
\hline
\end{tabular}

- Tab. 2 Screening bei der U2: Indikation zur Frenulotomie?

\begin{tabular}{|l|l|}
\hline Variable & Ausprägung \\
\hline \multirow{2}{*}{ Stillprobleme } & nein \\
\hline \multirow{2}{*}{ pathologische Peristaltik } & ja \\
\hline & $\begin{array}{l}\text { vollständig von vorne nach hinten, } \\
\text { beginnend an der Zungenspitze }\end{array}$ \\
\hline & $\begin{array}{l}\text { schwache Peristaltik oder Peristaltik } \\
\text { beginnt hinter der Zungenspitze }\end{array}$ \\
\hline $\begin{array}{l}\text { keine oder Peristaltik in umgekehr- } \\
\text { ter Richtung }\end{array}$ \\
\hline $\begin{array}{l}\text { ATLFF-Funktions-Score (Assess- } \\
\text { ment Tool for Lingual Frenulum } \\
\text { Function), Gesamtpunktzahl }\end{array}$ & $\geq 11$ Punkte \\
\hline & $<11$ Punkte \\
\hline
\end{tabular}

tung am ehesten von einer frühzeitigen Intervention. Liegen demnach gleichzeitig Stillprobleme sowie eine Ankyloglossie mit einer eingeschränkten Zungenmobilität vor, sollte nun eine Frenulotomie indiziert werden. Die Frenulotomie stellt bei Durchführung durch einen qualifizierten Arzt einen einfachen und sicheren Eingriff dar. Daher sollte im Zweifel bei einem funktionell bedeutsamen Zungenbändchen zu einer Frenulotomie geraten werden, auch wenn die Ankyloglossie nur einer von mehreren Faktoren ist, die den Stillerfolg behindern.

\section{FALLBEISPIEL}

\section{Fall 3}

Ein männliches Neugeborenes (36+1 T SSW) kommt per primärer Sectio auf die Welt. Die 30-jährige Erstgravida berichtet von Anfang an von starken Stillproblemen. Sie habe heftigste Schmerzen und habe sich das Stillen einfacher vorgestellt. Sie beginnt daher, das Neugeborene mit der Flasche zuzufüttern. In der Untersuchung der Mundhöhle im Rahmen der U2 fällt eine Ankyloglossie auf, eine Frenulotomie wird empfohlen. Die Mutter entscheidet sich gegen den Eingriff, da sie ihrem Neugeborenen keine Schmerzen zufügen möchte. Stattdessen entscheidet sie sich, am 5. Lebenstag abzustillen.

\section{Schlussfolgerungen}

Zur klinischen Relevanz des Zungenbändchens gibt es bisher keinen interdisziplinären und interprofessionellen Konsens. Während laut Messner et al. die Mehrzahl der befragten StillberaterInnen (69\%) glauben, dass ein Zungenbändchen häufig Stillprobleme verursacht, vertreten nur $10 \%$ der Pädiaterlnnen bzw. 30\% der HNOÄrztInnen diese Meinung [18].

Nach unserer Erfahrung hat das Zungenbändchen tatsächlich einen signifikanten Einfluss auf den Stillerfolg. Es konnte gezeigt werden, dass mehr als die Hälfte aller Neugeborenen mit Zungenbändchen Stillprobleme haben und dass ein Zungenbändchen ein relevanter Risikofaktor, insbesondere für schwere Stillprobleme, darstellt [13]. Deshalb sollte es im Rahmen von Stillförderungsmaßnahmen in den Geburtskliniken und in der ambulanten postpartalen Betreuung von Mutter-KindPaaren als mögliches Stillhindernis berücksichtigt werden.

Um eine Ankyloglossie als Stillhindernis zu beseitigen, sollte eine frühzeitige Diagnose erfolgen. Die Einführung eines allgemeinen Screenings auf Zungenbänd- 
chen und weiterer Stillhindernisse wäre daher ein wichtiger Schritt, um den Stillerfolg zu verbessern und ein längeres ausschließliches Stillen zu fördern.

Dabei könnte unser Screeningtool zur Identifizierung von Stillproblemen eingesetzt werden, da es neben dem Zungenbändchen-spezifischen ATLFF-Score auch diverse weitere Stillhindernisse integriert und nach unseren Daten bei Säuglingen mit Ankyloglossie eine höhere Sensitivität und Spezifität aufweist als die alleinige Anwendung des ATLFF-Scores. Durch eine feste Aufnahme des Screenings in die Vorsorgeuntersuchungen U1 und U2 könnten frühzeitig Probleme identifiziert werden.

Es bleibt zu überprüfen, ob ein Teil der Risikofaktoren durch eine konsequentere Stillberatung kompensiert werden kann. In indizierten Fällen sollte bei der U2 eine Frenulotomie initiiert werden, die bei qualifizierter Durchführung eine einfache, sichere und erfolgreiche Therapie darstellt.

Zukünftig braucht es für mehr Klarheit in der kontroversen Diskussion um die Relevanz und Therapie der Ankyloglossie eine standardisierte, klar verständliche und einfach anzuwendende Definition. Des Weiteren sind qualitativ hochwertige randomisiert-kontrollierte Studien unbedingt erforderlich, um einen Konsens in Bezug auf Indikation, Zeitpunkt und Methode der operativen Therapie zu erreichen.

\section{KERNAUSSAGEN}

- Eine Ankyloglossie bezeichnet eine angeborene Entwicklungsstörung der Zunge, bei der ein zu straffes oder zu kurzes Zungenbändchen die Zunge am Mundboden fixiert. Dieses setzt häufig zu weit vorne an der Zungenspitze und an der Zahnleiste an und behindert so die Beweglichkeit der Zunge.

- Stillprobleme werden häufig durch ein gestörtes Saugverhalten des Neugeborenen verursacht. Eine eingeschränkte Zungenbeweglichkeit kann den korrekten Saugmechanismus behindern.

- Eine Ankyloglossie ist ein relevanter, behandelbarer Risikofaktor für ein nicht erfolgreiches Stillen und sollte daher als solcher berücksichtigt werden.

- Zur Bewertung der Ankyloglossie kann der ATLFF-Score mit besonderer Berücksichtigung der Zungenfunktion empfohlen werden. Eine Gesamtpunktzahl des ATLFF-FunktionsScores $<11$ Punkte sowie eine pathologische Peristaltik der Zunge sprechen dabei für eine relevante Funktionsstörung.

- Neben dem Zungenbändchen-spezifischen ATLFF-Score spielen auch weitere Risikofaktoren wie die fehlende Stillerfahrung, Untergewicht oder Frühgeburtlichkeit des Neugeborenen eine wichtige Rolle bei ausbleibendem Stillerfolg. Diese Faktoren sollten daher in den pädiatrischen Vorsorgeuntersuchungen ebenso erfasst werden.

- Eine Stillberatung sollte als erste und wichtigste Maßnahme bereits bei Vorliegen von Risikofaktoren eingeleitet werden.

- Bei einer Ankyloglossie mit relevanter Einschränkung der Zungenbeweglichkeit und Stillproblemen sollte eine Frenulotomie indiziert werden. Diese stellt bei qualifizierter Durchführung eine einfache, sichere und erfolgreiche Therapie dar.

\section{Schlüsselwörter}

Ankyloglossie, Zungenbändchen, Stillprobleme

Interessenkonflikt

Erklärung zu finanziellen Interessen

Forschungsförderung erhalten: nein; Honorar/geldwerten Vorteil für Referententätigkeit erhalten: nein; Bezahlter Berater/interner Schulungsreferent/Gehaltsempfänger: nein; Patent/Geschäftsanteile/Aktien (Autor/Partner, Ehepartner, Kinder) an Firma (Nicht-Sponsor der Veranstaltung): nein; Patent/Geschäftsanteile/Aktien (Autor/Partner, Ehepartner, Kinder) an Firma (Sponsor der Veranstaltung): nein.

Erklärung zu nichtfinanziellen Interessen

Die Autorinnen/Autoren geben an, dass kein Interessenkonflikt besteht. 


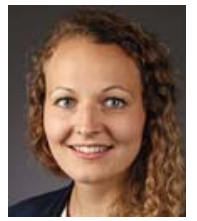

Sara-Maria Simon

Dr. med. Jahrgang 1991. 2010-2017 Studium der Humanmedizin an der Albert-LudwigsUniversität Freiburg. 2017-2019 Assistentin in Weiterbildung in der Abteilung für Kinderund Jugendmedizin am St. Josefskrankenhaus Freiburg, seit 2019 am Zentrum für Kinderund Jugendmedizin, Universitätsklinikum Freiburg.

\section{Korrespondenzadresse}

Dr. med. Sara-Maria Simon

Zentrum für Kinder- und Jugendmedizin

Universitätsklinikum Freiburg

Mathildenstr. 1

79106 Freiburg

Deutschland

E-Mail: sara-maria.simon@uniklinik-freiburg.de

Wissenschaftlich verantwortlich gemäß Zertifizierungsbestimmungen

Wissenschaftlich verantwortlich gemäß Zertifizierungsbestimmungen für diesen Beitrag ist Dr. med. Sara-Maria Simon, Freiburg.

Literatur

[1] Francis DO, Krishnaswami S, McPheeters M. Treatment of Ankyloglossia and Breastfeeding Outcomes: A Systematic review. Pediatrics 2015; 135: e1458-e1466

[2] O'Callahan C, Macary S, Clemente S. The effects of officebased frenotomy for anterior and posterior ankyloglossia on breastfeeding. Int J Pediat Otorhinolaryngol 2013; 77: 827 832

[3] Hentschel R. Breastfeeding problems should be the only relevant criteria for deciding whether to carry out a frenotomy in infancy. Acta Paediat 2018; 107: 1697-1701

[4] Bath-Balogh M, Fehrenbach M]. Illustrated dental embryology, histology, and anatomy. 4th edn. Missouri: Elsevier Health Sciences; 2016

[5] Victora CG, Bahl R, Barros A] et al. Breastfeeding in the 21st century: epidemiology, mechanisms, and lifelong effect. Lancet 2016; 387: 475-490

[6] Brettschneider A-K, von der Lippe E, Lange C. Stillverhalten in Deutschland - Neues aus KiGGS Welle 2. Bundesgesundheitsbl Gesundheitsforsch Gesundheitsschutz 2018; 61: 920-925

[7] Brettschneider A-K, Weikert C, Abraham K et al. Stillmonitoring in Deutschland - Welchen Beitrag können die KiGGS-Daten leisten? Journal of Health Monitoring 2016: doi:10.17886/RKI-GBE-2016-038

[8] World Health Organization, Unicef. Breastfeeding and maternal medication: recommendations for drugs in the eleventh WHO model list of essential drugs. 2002: Im Internet (Stand: 05.08.2020): http://apps.who.int/iris/bitstream/ handle $/ 10665 / 62435 / 55732$. pdf? sequence $=1$ \&isAllowed=y
[9] Carlsen SM, Jacobsen G, Vanky E. Mid-pregnancy androgen levels are negatively associated with breastfeeding. Acta Obstet Gynecol Scand 2010; 89: 87-94

[10] Dewey KG, Nommsen-Rivers LA, Heinig M] et al. Risk factors for suboptimal infant breastfeeding behavior, delayed onset of lactation, and excess neonatal weight loss. Pediatrics 2003; 112: 607-619

[11] Srinivasan A, Dobrich C, Mitnick $\mathrm{H}$ et al. Ankyloglossia in breastfeeding infants: the effect of frenotomy on maternal nipple pain and latch. Breastfeed Med 2006; 1: 216-224

[12] Dollberg S, Botzer E, Grunis E et al. Immediate nipple pain relief after frenotomy in breast-fed infants with ankyloglossia: a randomized, prospective study. J Pediat Surg 2006; 41: $1598-1600$

[13] Schlatter S-M, Schupp W, Otten J-E et al. The role of tonguetie in breastfeeding problems - A prospective observational study. Acta Paediat 2019; 108: 2214-2221

[14] Rasenack R, Schneider C, Jahnz E et al. Einflussfaktoren auf die Stilldauer im Freiburger Geburtenkollektiv (FreiStill). Geburtsh Frauenheilkd 2012; 72: 1-7

[15] Woolridge MW. The „anatomy“of infant sucking. Midwifery 1986; 2: 164-171

[16] Suter VGA, Bornstein MM. Ankyloglossia: facts and myths in diagnosis and treatment. J Periodontol 2009; 80: 12041219

[17] Ballard JL, Auer CE, Khoury JC. Ankyloglossia: assessment, incidence, and effect of frenuloplasty on the breastfeeding dyad. Pediatrics 2002; 110: e63-e63

[18] Messner AH, Lalakea ML. Ankyloglossia: controversies in management. Int J Pediat Otorhinolaryngol 2000; 54: 123131

[19] Karall D. Das zu kurze Zungenband. Pädiat Pädol 2014; 49 28-33

[20] Griffiths DM. Do tongue ties affect breastfeeding? J Human Lactat 2004; 20: 409-414

[21] Hazelbaker AK. The Assessment tool for lingual frenulum function [master's thesis]. Pasadena (CA): Pacific Oaks College; 1993

[22] Lalakea ML, Messner AH. Ankyloglossia: does it matter? Pediat Clin North Am 2003; 50: 381-397

[23] Fiorotti RC, Bertolini MM, Nicola JH et al. Early lingual frenectomy assisted by $\mathrm{CO} 2$ laser helps prevention and treatment of functional alterations caused by ankyloglossia. Int J Orofac Myol 2004; 30: 64-71

[24] Canadian Agency for Drugs and Technologies in Health. Frenectomy for the correction of ankyloglossia: a review of clinical effectiveness and guidelines. CADTH Rapid Response Reports; 2016: Im Internet (Stand: 05.08.2020): http:// www.ncbi.nlm.nih.gov/books/NBK373454/

[25] Berry ], Griffiths M, Westcott C. A double-blind, randomized, controlled trial of tongue-tie division and its immediate effect on breastfeeding. Breastfeeding Medicine 2012; 7: 189-193

[26] Emond A, Ingram J, Johnson D et al. Randomised controlled trial of early frenotomy in breastfed infants with mild-moderate tonque-tie. Arch Dis Childhood Fetal Neonat Edn 2014; 99: F189-F195

[27] Hogan M, Westcott C, Griffiths M. Randomized, controlled trial of division of tongue-tie in infants with feeding problems. J Paediat Child Health 2005; 41: 246-250

[28] O'Shea JE, Foster JP, O'Donnell CP et al. Frenotomy for tongue-tie in newborn infants. Cochrane Database of Systematic Reviews 2017: doi:10.1002/14651858.CD011065.pub2 
[29] Academy of Breastfeeding Medicine Protocol Committee. ABM clinical protocol 11: guidelines for the evaluation and management of neonatal ankyloglossia and its complications in the breastfeeding dyad. 2004: Im Internet: https:// abm.memberclicks.net/assets/DOCUMENTS/PROTOCOLS/ 11-neonatal-ankyloglossia-protocol-english.pdf
Bibliografie

Neonatologie Scan 2021; 10: 59-71

DOI 10.1055/a-1019-2774

ISSN 2194-5462

(c) 2021. Thieme. All rights reserved.

Georg Thieme Verlag KG, Rüdigerstraße 14,

70469 Stuttgart, Germany 


\section{Punkte sammeln auf CME.thieme.de}

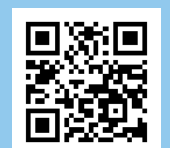

Diese Fortbildungseinheit ist in der Regel 12 Monate online für die Teilnahme verfügbar.

Den genauen Einsendeschluss finden Sie unter https://cme.thieme.de.

Sollten Sie Fragen zur Online-Teilnahme haben, finden Sie unter https://cme.thieme.de/hilfe

eine ausführliche Anleitung. Wir wünschen viel Erfolg beim Beantworten

der Fragen!

Unter https://eref.thieme.de/CXDWDBK oder über den QR-Code kommen Sie direkt zur Startseite des Wissenstests.

VNR 2760512021160213561

\section{Frage 1}

Welcher dieser Faktoren stellt kein relevantes Stillrisiko dar?
A Frühgeburtlichkeit
B fehlende Stillerfahrung
C Untergewicht
D Spontangeburt
E Ankyloglossie

\section{Frage 2}

Wodurch ist die Epidemiologie der Ankyloglossie gekennzeichnet?

A Weibliche Neugeborene sind häufiger betroffen.

B Die Inzidenz einer Ankyloglossie variiert in der wissenschaftlichen Literatur aufgrund fehlender diagnostischer Standards.

C Schwere Stillprobleme treten bei Ankyloglossie in maximal $10 \%$ der Fälle auf.

D Die Inzidenz einer Ankyloglossie liegt zwischen 20 und $85 \%$.

E Stillprobleme treten bei Neugeborenen mit Ankyloglossie nicht häufiger auf als bei Neugeborenen ohne Ankyloglossie.

\section{Frage 3}

Welche Stillprobleme werden nicht direkt mit einer Ankyloglossie in Verbindung gebracht?
A wunde Brustwarzen
B Probleme beim Andocken
C schlechter Milchtransfer, Gedeihstörungen
D unzureichende mütterliche Milchproduktion
E Brustschmerzen

\section{Frage 4}

Bei welchem ATLFF-Kriterium (ATLFF=Assessment Tool for Lingual Frenulum Function) zum Erscheinungsbild des Zungenbändchens wird die volle Punktzahl vergeben, das heißt der Befund ist normal?

A eine Länge $>1 \mathrm{~cm}$ bei angehobener Zunge

B eine herzförmige Zungenspitze

C ein Ansatz des Zungenbändchens unter der Oberkante der Zahnleiste

D ein Ansatz des Zungenbändchens, der mehr als $50 \%$ der Mittellinie der Zungenunterseite beansprucht

E mäßige Elastizität des Zungenbändchens

\section{Frage 5}

Einer der folgenden Parameter gehört nicht zum ATLFF-Funktions-Score. Welcher?

A Lateralisation der Zunge

B Zurückschnalzen der Zunge beim Saugen

C Peristaltik der Zunge

D rhythmisches Saugen

E Anheben der Zunge

\section{Frage 6}

Welche Aussage zum korrekten Saugmechanismus ist falsch?

A Der Saugreflex wird durch Berührung des Gaumens ausgelöst.

B Während des Saugens wird lediglich der vorderste Teil der Brustwarze in die Mundhöhle aufgenommen.

C Ein nutritives Saugen besteht aus einem rhythmischen Wechsel zwischen Saugen und Auspressen der Brustwarze.

D Eine gute Zungenbeweglichkeit ist eine Voraussetzung für den korrekten Saugmechanismus.

E Ein korrektes Saugverhalten erfordert die Koordination aus Saugen, Schlucken und Atmen. 


\section{Punkte sammeln auf CME.thieme.de}

Fortsetzung ...

\section{Frage 7}

Bei welchem Befund werden beim ATLFF-Funktions-Score Punkte abgezogen?

A Die Zungenspitze hebt sich bis zur halben Höhe des Mundes.

B Die peristaltische Welle beginnt hinter der Zungenspitze.

C Die Zunge breitet sich vor der Bildung eines Saugschlusses vollständig aus und wird dünn.

D Der Saugschluss ist stark, mit ganzem Zungenrand.

E Die Zungenspitze wird über die Unterlippe herausgestreckt.

\section{Frage 8}

Welches Kriterium gehört nicht zum Screeningtool im Rahmen der U1 zur Evaluierung von Stillproblemen bei Ankyloglossie?
A Erfassung von Stillproblemen wie z. B. wunde Brustwarzen oder Brustschmerzen
B Untersuchung des Zungenbändchens
C Erfassung des Geburtsmodus
D Erhebung der Milchmenge
E Erfassung des Gestationsalters

\section{Frage 9}

Welche Aussage zur Frenulotomie bei Ankyloglossie ist richtig?

A Eine Frenulotomie ersetzt eine frühzeitige Stillberatung.

B Eine Frenulotomie ist eine einfache und sichere Therapieoption, die von jedem Arzt durchgeführt werden kann.

C Eine Frenulotomie sollte unter Allgemeinanästhesie durchgeführt werden.

D Bei einer Ankyloglossie mit gleichzeitig bestehenden Stillproblemen kann eine Frenulotomie indiziert sein.

E Eine Frenulotomie sollte bei jedem auffälligen Zungenbändchen zur Vorbeugung von späteren Sprachproblemen durchgeführt werden.

\section{Frage 10}

Ein männliches Neugeborenes wird in der 40. Schwangerschaftswoche spontan entbunden. Die Mutter ist Erstgravida, bei der U1 wird ein auffälliges Zungenbändchen festgestellt. Was ist die erste und wichtigste Maßnahme?
A Erhebung des ATLFF-Funktions-Scores
B Durchführung einer Stillberatung
C Durchführung einer Frenulotomie
D keine Maßnahme, bis Stillprobleme auftreten
E frühzeitiges Zufüttern mit Flaschennahrung 